\title{
Cognitive and social factors in the development of infants with Down syndrome
}

\author{
Derek G. Moore ', John M. Oates ${ }^{2}$, R. Peter Hobson ${ }^{3}$ and Julia Goodwin ' \\ ' Department of Psychology, University of East London, ${ }^{2}$ Centre for Human Development and Learning, Open \\ University, ${ }^{3}$ Developmental Psychopathology Research Unit, Tavistock Clinic \& University College London
}

\begin{abstract}
Infants and young children with Down syndrome can be engaging and affectionate. It seems that in the early months of life their personal relations may be relatively 'spared' the effects of limitations in their capacities for information-processing. Yet how far is this the case as development proceeds? In this paper we discuss some ways in which social and cognitive development interact and mutually influence one another over the first year or so of life, and present preliminary findings from a longitudinal study of infants with and without Down syndrome. The evidence suggests that the development of 'triadic' (person-person-world) social interactions may be affected by limited information-processing capacities in infants with Down syndrome, through a complex socially-mediated developmental trajectory.
\end{abstract}

Keywords: Down syndrome, infant attention, information processing, mother-infant interaction, joint attention, transactional processes

\section{Introduction}

Delay in the development of cognitive capacities is a primary consequence of Down syndrome. Despite such cognitive delay, however, young children with Down syndrome can be empathic, affectionate and engaging (Wishart \& Pitcairn, 2000). This might be taken to suggest that there are pre-specified, independent, 'domain specific' pathways for some aspects of social and cognitive development. Yet if one adopts a perspective that overemphasises modularisation of function there is a danger that one may underestimate the complex transactional processes that occur between domains in social and cognitive development (see Karmiloff-Smith, 1998). We need to consider not only the possibility of relatively independent 'domain specific' developmental trajectories for some social and cognitive capacities, but also to work out the extent to which cognitive and social development capacities, even those that may appear initially to develop relatively independently of each other, come to interact with and mutually influence one another, and lead to unique developmental outcomes. To this end we have conducted a detailed longitudinal study of a cohort of infants with and without Down syndrome and their mothers, following their progress from an early age. We have assessed children with Down syndrome at six monthly intervals from 6 through to 18 months, and contrasted their development with a control group of typically developing infants seen at 4, 7 and 10 months.
Our aim was to study how social and cognitive capacities combine to create characteristic forms of infant-parent interaction in families of children with Down syndrome, and consider how these forms of interaction, in turn, regulate and influence other aspects of their social and cognitive development. This approach may help to explain the emergence of subtle differences as well as similarities in the social and cognitive capacities of people with Down syndrome when compared to typically developing children (Chapman \& Hesketh, 2000). This paper gives an overview of some findings emerging from this study and outlines some of the methodological and conceptual issues that we have begun to address.

\section{Difficulties in measuring cognitive capacities}

For many years the dominant view was that 'cognition' could be construed largely in terms of children's progressive construction of knowledge based on their own active interaction with the world (Piaget, 1954). This view was challenged by psychologists adopting a so-called Vygotskian perspective (e.g. Bruner, 1975; Wertsch, 1985; Rogoff, 1990). These theoreticians emphasised the role that social interaction and context plays in the co-construction of a child's knowledge. Two issues relevant to our research emerge from these theoretical views. Firstly, these perspectives remind us that the functions and structure of the 
brain are not entirely predetermined, they are also dependent on experience and active interaction with the world. Secondly, this reminds us that experiences are not purely a consequence of the activities of the individual. An individual's neuro-cognitive development is also determined by the instruction and scaffolding of others. At the very least others help to regulate the focus of the individual's attention and assign significance to information in the external world through social referencing and other 'person-personworld' interaction (see Hobson, 2002). Hence, particularly early in life, to adequately characterise neuro-cognitive development we must consider the social context in which the infant is immersed. Secondly, this approach has highlighted limitations in tests of cognitive functioning and the importance, when testing children's individual cognitive capacities, of considering the influence of the social context in which a test occurs (Light, 1986). This does not mean that a relatively 'pure' cognitive test cannot be devised, but it must be devised taking into account the influence on performance of the social context.

Thus, to assess interactions and transactions between cognitive and social domains we need to have measures of an individual's level of cognitive functioning that have allowed for social influences. We also need to define clearly what 'cognitive' functions are. Early cognitive ability has been characterised as the development of abilities to form and utilise representations of the world and to develop conceptual models or theory-like constructs. Some cognitive tests of babies, correspondingly, look for abilities to represent hidden objects or to distinguish or match different objects based on their conceptual meaning, for example in terms of category membership. Once the child has passed infancy it becomes possible to look at the development of capacities to represent the world through language.

Cognitive abilities also implicate capacities to direct and control one's attention and to regulate one's thinking and behaviour. These capacities for attending to the world, storing and encoding experience and representations, creating and executing planned actions (means-ends), and controlling and inhibiting responses, are often referred to as information-processing capacities. It has been argued that information-processing capacities may represent the most fundamental, purest and best estimate of mental ability and that impairments in information processing may underlie delays in the development of representational capacities (Zelazo \& Stack, 1997). This view is supported by evidence that estimates of information processing capacities in infants appear to be better predictors of future IQ than standardised tests of developmental progress (see Slater, 1995). In infancy, information processing is most often assessed by studying infants' abilities to control and direct their attention to visual stimuli. Approaches include the repeated presentation of pairs of interesting stimuli on computer or television screens and the recording of infants' spontaneous or novelty preferences. Alternatively, one can present infants with a single stimulus and assess the rate at which infants become 'used to' the stimulus (habituation) and the amount of recovery of attention they show when presented with a new stimulus (dis-habituation).

Colombo (2001) has proposed that the development of four separate neural substructures underpins the development of infants' attention, with each structure responsible for a different aspect of attentional control. These are: Alertness or anticipatory readiness for stimuli which is modulated by brainstem activities; Spatial Orienting, the shifting of attention to and from particular stimuli under the control of posterior brain systems; Attention To Object Features, systems for analysing the visual properties of stimuli that will lead to their identification, determined by pathways in the occipital and temporal cortex; and Endogenous Attention, volitional capacities for directing, holding or inhibiting the direction of attention mediated by the frontal lobes. This function being the last to develop in typically developing infants. These sub-systems seem to represent important components of information processing and it is proposed that these may be the basis on which cognitive development progresses.

There is evidence that children with Down syndrome differ from typically developing infants in the development and deployment of their attentional resources and have known neurological impairments. For example, differences have been found both in utero and in early infancy, in the rate of habituation of infants with Down syndrome (Hepper \& Shaidullah, 1992). Also infants with Down syndrome show different patterns of visual preference in early infancy (Miranda \& Fantz, 1973; Miranda, 1976). Correspondingly, when performing information processing tasks, people with Down syndrome show differences in the amplitude of cerebral event-related potentials (Karrer, Wojtascek \& Davis, 1995). These differences in attentional performance and in event-related potentials are likely to be a consequence of differences in the structure and development of the frontal lobes and cortex (i.e. Logdberg \& Brun, 1993; Takashima, Becker, Armstrong \& Chan, 1981). Karrer, Karrer, Bloom, Chaney and Davis (1998) have proposed that children with Down syndrome may have particular impairments in 'frontal' processing that may affect a range of inhibitory processes (corresponding to Colombo's endogenous attention). However, more detailed examinations of component attentional processes are required. Focussing on different attentional systems in the terms outlined by Colombo (2001), may allow us to describe the unique developmental profile of infants with Down syndrome in a more fine-grained way. We may be able to establish whether specific components are impaired or spared in infants with Down syndrome or whether infants with Down syndrome have a more global information-processing deficit. For example Zelazo and Stack (1997) suggest that inhibitory problems may be consequence of slower overall processing and not a product of a specific impairment in frontal processes.

In our studies we have presented infants with a series of static and moving stimuli on computer screens. The primary intention was to look at capacities for visual dis- 
crimination between meaningful stimuli, requiring what Colombo (2001) refers to as 'attention to object features' but the tasks also yielded measures relating to other aspects of attention. Zelazo and Stack (1997) suggest that sequential stimuli may be the best visual stimuli to use for assessing attentional capacities. Therefore, of particular interest, were infants' responses during a task involving the repeated presentations of animations of circles depicting 'billiard ball', causal and non-causal collisions (based on Leslie \& Keeble, 1987). The first test condition was a non-causal event and involved one circle appearing from one side of the screen and moving towards another circle located in the centre. The first circle stopped before reaching the central ball. The second circle then moved off and disappeared off the other side of the screen (a non-caused action). This event was repeated until the infants habituated. A number of measures of attention were collected. These were: the total duration of looking; the length of longest look; the number of looks; the number of complete stimulus events seen; the total duration of looking away and the latency of orienting to the first presentation of the event. Surprisingly the last two measures are not normally reported in habituation studies but are the most likely to provide useful information regarding inhibitory attentional processes in infants with Down syndrome (Oates, Moore \& Hobson, 1997; Moore, Oates, Goodwin \& Hobson, 2000).

Factor analysis of these measures in our cohort of typically developing infants and infants with Down syndrome suggested that these measures might indeed be tapping into separate attentional processes. The analysis yielded a threefactor solution. These factors seem to map onto three of the components of attention identified by Colombo. Duration of looking and the length of the longest look loaded together and seemed to represent an alertness factor; the number of looks and number of complete stimulus events seen loaded together and indicated spatial orienting; and the duration of looking away between looks and the latency of orienting to the first presentation of a stimulus seemed to be a measure of endogenous attention (Moore et al., 2000).

We examined the performance of the infants with Down syndrome when 6 months old, and compared their performance to our control group of typically developing infants at 4 months of age, when considered comparable in general developmental level (see Rauh, Schellhas, Goeggerle \& Muller, 1996). Results revealed that the infants with Down syndrome, showed significantly lower alertness (smaller duration of looking) and endogenous attention (slower latency and longer looks away) but were equivalent in spatial orienting (Moore et al., 2000). Thus, these component measures of attention seem to capture specific differences and not just delays in cognitive functioning between children with Down syndrome and typically developing children. These differences may not be picked up by more traditional cognitive tests, and while differences in alertness may be picked up by traditional habituation measures, endogenous attention may only be possible to index during habituation by examining patterns of latency and looking away'. One particular advantage of this approach is that the measurement of attentional process is not so dependent on establishing a social rapport with the infant. Thus it may be a more profitable way to assess the 'pure' cognitive abilities of children with Down syndrome independently of social competence (Zelazo \& Stack, 1997).

While visual attention tasks may prove to be particularly useful for testing cognitive functioning in infants, they are rarely used for this purpose. Instead developmental tests such as Bayley Scales are often used to assess cognitive level (Bayley, 1969; 1993). The Bayley scales consist of a number of tasks devised to assess the competence of babies at each month of development, and to provide an indication of whether the babies have achieved a typical developmental level. Some tasks may be administered only to younger or older infants. The scale includes Piagetian object-search and means-ends tasks designed to assess the development of representational abilities, as well as tasks assessing fine motor skills, visual perceptual processing, planning abilities, memory, social abilities and language. By looking at performance across the whole range of tasks administered, the Bayley scales deliver scores on four developmental 'facets': cognitive, social, language and motor.

For this project, the central problem with using this scale for extracting a 'pure' cognitive measure was that all the tasks of the Bayley scales, including those that go to make up the cognitive facet score, involve a social interaction between tester and infant, confounding social with cognitive competence. Some aspects of task performance may be determined not only by an infant's cognitive abilities but also by the capacity of child and tester to develop a rapport, and thereby to establish scaffolding. There are also additional problems for researchers of infants with Down syndrome. The tasks intended to be indicators of cognitive level also rely on motor manipulation and language to demonstrate competence. In fact, many of the individual tasks contribute to scores on the motor, language and cognitive facets. This is not a problem when using the scales as a clinical tool to identify the presence of atypical development, as the identification of a delay or difference in performance is the main objective, not the identification of the source of the performance deficit. However, where there are known specific language and motor delays, as is often the case for children with Down syndrome, these language and motor delays may obscure other cognitive competencies and lead to an underestimation of 'pure' cognitive abilities.

For this reason, we have made an attempt to extract out from the Bayley scales a collection of those tasks where the social, motor and language components have a reduced influence on the expression of cognitive competence. The aim has been to create a shorter, relatively pure, cognitive scale (Goodwin, Oates \& Moore, 2000). With this shortform, a score of cognitive achievement can be constructed by considering the number of tasks passed at each age. This short-form can then be used to assess the relationships, within-groups, between cognitive and social abilities. While still in development, the measure has been useful 
as a means of establishing the equivalence of our groups of infants with and without Down syndrome in their cognitive functioning at each data sweep (Goodwin et al., 2000). Using this technique we have been able to confirm that the cognitive level of our infants with Down syndrome when 6,12 and 18 months old is equivalent to that of our control typically developing infants when 4, 7 and 10 months old. This supported our initial estimates of likely cognitive levels based on Rauh et al. (1996).

In summary, we have established, using a short form of the Bayley scales, that it may be possible to measure some aspects of cognitive functioning relatively independently of social, motor and language capacities. This also established more clearly the cognitive equivalence of our cohorts of typically developing infants and infants with Down syndrome at each data collection point. We have also been able, using habituation tasks, to measure important differences in attentional capacities between the cohorts that may underpin delays in cognitive development. It may, for example, prove that these attentional differences underpin the different profiles of errors and instability in cognitive performance of infants with Down syndrome noted, for example, by Morss (1983) and Wishart and Duffy (1990).

\section{Cognitive influences on social development}

How do these unique profiles of cognitive delay and difference influence social development in children with Down syndrome? In particular, to what extent and by what mechanisms do attentional differences influence the development of social understanding? One argument might be that just because infants with Down syndrome have problems with attending to the world in general it is not inevitable that they will be impaired in attending to people. Indeed, it is plausible to argue that some early processes used for interpreting people's faces and extracting social meanings, such as their emotions, may use specialised, dedicated, modular like, low-level neurological architecture. At least in early development, these may operate independently of the neurological systems responsible for general attention detailed by Colombo (see Moore, Hobson \& Anderson, 1995). It is also possible that these systems may be relatively spared in people with intellectual deficits and, because of this, people with attentional problems and intellectual deficits may be able, in early infancy, to develop relatively typical social relationships.

Recent evidence partly supports this view, as it seems that young children with Down syndrome perform similarly to typically developing children matched for mental age on emotion recognition tasks (Kasari, Freeman \& Hughes, 2001), and Heimann, Ullstadius and Swerlander (1998) report spared imitative abilities. However, there is also evidence that older children with Down syndrome perform poorly on emotion recognition tasks relative both to mental age equivalent typically developing children and to people with general learning difficulties (Wishart \& Pitcairn, 2000). The source of these performance deficits remains to be established. Perhaps they do reflect a basic impairment in emotion recognition. However it may be that primary emotional capacities are intact, and it is the expression of these that is constrained by the reduced information-processing capacities of the infants with Down syndrome. This difference may not controlled for by matching on measures such as the Bayley scales. If infants with Down syndrome are slower or more inconsistent in orienting and responding then this may well impair their ability to perform emotion recognition tasks (Moore, 2001) and affect their responses to people in social interactions. Even if basic sensitivities to emotional patterning are preserved, the differential responses of infants with Down syndrome may create a different quality of social interaction that will lead to different developmental outcomes (Richard, 1986).

In order to develop a fuller picture of the development of infants with Down syndrome we need to explore these transactional processes across time. Specifically we need to assess: infants' constituent attentional capacities in non-social situations; infants' attention and behaviour within social interactions; the effect of their behaviour on caregivers; and the processes by which different forms of mother-infant interaction, in turn, influence the social and cognitive development of the child. As outlined above, there is already evidence that infants with Down syndrome demonstrate attentional problems in non-social situations. There is also some evidence that infants show different attentional profiles in social interactions.

For example, in a longitudinal study of five infants with Down syndrome and a control group of typically developing infants from 1 to 6 months, Berger and Cunningham (1981) reported that during a face-to-face interaction with their mother typically developing infants showed the greatest levels of eye-contact between 2 and 4 months of age. After four months of age this level of eye contact then falls off. In contrast, infants with Down syndrome showed a delayed but steady increase in percentage eye-contact over the course of the first six months reaching the levels of 4-month-old typically developing infants by around six months of age. Infants with Down syndrome did not then show a fall off in looking and seem to maintain higher levels of looking to their mothers. Also, their looking was characterised by shorter lengths of individual eye-contact episodes.

While infants with Down syndrome seem to differ in the time they spend looking to the mother, both typically developing infants and infants with Down syndrome show a similar reduction in eye contact when their mother ceases interacting with them during a 'still-face' period. This suggests that the infants in each group have similar emotional reactions to the unresponsive behaviour of the mother. Berger and Cunningham (1986) also reported that infants with Down syndrome showed reduced smiling during face-to-face interactions. Similar findings were reported by Legerstee and Bowman (1989) and by Crown, Feldstein, Jasnow, Beebe and Jaffe (1992). 
Similarly despite delays in the achievement of levels of eye-contact, and shorter individual eye-contact episodes in face-to-face interactions, Gunn, Berry and Andrews (1982) reported that 6- to 9-month-old infants with Down syndrome spent more total time during play looking at their mother than at objects. This looking was nearly twice that of typically developing infants of the same chronological age. Landry and Chapieski (1990) studied 6-month-old infants with Down syndrome and found that infants with Down syndrome looked more to the mother and less at a toy even compared to pre-term infants matched for mental age. This also occurred even when the mothers were not actively involved in play. Thus, early in development, infants with Down syndrome may be exhibiting less eye contact but actually looking for longer amounts of time at their mothers than chronological age- and mental agecomparable control infants.

How does the behaviour of the infants with Down syndrome influence maternal behaviour? Is there any evidence that the behaviour of mothers of infants with Down syndrome is different from that of mothers of typically developing infants? Hyche, Bakeman and Adamson (1992) reported that mothers who had brought up children with Down syndrome were more sensitive to the communicative cues captured on videotape of children with Down syndrome than mothers of typically developing infants. However, mothers of infants with Down syndrome, despite being more sensitive to the cues of infants with Down syndrome, still found infants with Down syndrome of around 7 months of age more difficult to 'read' than typically developing infants of the same age. How would this influence their behaviour? Buckhalt, Rutherford and Goldberg (1978) reported that their sample of babies with Down syndrome, ranging in age from 9 to 18 months, smiled and vocalised less and that, correspondingly, the mothers of infants with Down syndrome spoke to their infants at a significantly faster rate than mothers of typically developing infants. This suggests that the form of mother-infant interaction for Down syndrome dyads may come to differ in some important respects to that of typical dyads.

Thus there is evidence that infants with Down syndrome differ in their attentional capacities and propensities and this is evident in their behaviour in both social and nonsocial situations. There is also some evidence that maternal behaviour may differ as a consequence of these differences in attentional capacities. However, there have been no studies that have concurrently measured social and non-social attentional factors and established their role in influencing maternal interactional style. This is necessary if one is attempting to develop a transactional model of the development of infants with Down syndrome.

In our study, we examined mother-infant interactions during early infancy (at 4 months for typically developing children and 6 months for children with Down syndrome). We used the still-face paradigm of Tronick, Als, Adamson, Wise and Brazelton (1978) to assess the quality of early mother-infant interactions. In the first phase of the proce- dure mother and infants engaged in face-to-face interaction for three minutes. In the second phase the mother was asked, when a signal was given, to display a passive face and not to respond to the infant for up to 90 seconds, but less if the infant became distressed. In the final phase the mother and infant re-engaged in their normal interaction for 3 minutes.

We found that our sample of infants with Down syndrome responded similarly to typically developing infants. The groups showed the same percentage amount of looking and smiling to the mother during the first and final phases, and a similar decrement in looking and smiling when a still face was displayed. They did differ however in the amount of fussing, with infants with Down syndrome showing less fussing during the still face event than typically developing infants (Oates, Moore, Goodwin, Hobson \& Reynolds, 2002). These findings do not concur with those of Berger and Cunningham (1981) and this may be due to different methods, but an additional important finding emerged from this task that may also help explain these contrary findings.

While, in general, the infants with Down syndrome did not appear to behave differently to typically developing infants, the mothers did. We found that the mothers of infants with Down syndrome in our sample differed from typically developing mothers in their behaviour during the interaction phases when rated on the mother-infant interaction scales devised by Murray, Fiori-Cowley, Hooper and Cooper (1996). Using Principal Component Analysis we found that these 14 scales reduce to three meaningful factors. We found that the mothers of infants with Down syndrome significantly differed from the mothers of typically developing infants on two of these factors. Mothers of children with Down syndrome scored significantly higher than the mothers of typically developing infants on the factor comprised of ratings of 'effort to engage', 'warmth', 'happiness', 'absorption in their infant', etc. - the factor we have labelled 'warmth'. However, alongside this positive interaction quality the mothers also differed on the factor we have labelled 'forcefulness' derived from ratings of 'intrusiveness', 'sensitivity', and 'responsivity', with mothers of Down syndrome being rated as less sensitive, less responsive and more intrusive (Oates, Moore, Goodwin, Hobson, \& Reynolds, 1998; Oates, et al., 2002).

We have come to call this combined style of interaction 'forceful warmth', and have found that the degree to which the mothers of infants with Down syndrome demonstrated 'forceful warmth' was strongly associated with the infants' endogenous attention measured on the non-social visual attention task described earlier. We hypothesise that the reason the infants with Down syndrome in our cohort showed similar levels to the typically developing infants in attention and smiling during the face-to-face interaction (in contrast to the findings of Berger \& Cunningham, 1981, 1986) is that the mothers were working to increase their infants' responsivity to them by showing greater warmth. While this style may be positive in terms of engaging the 
infants, the mothers may also demonstrate a corresponding reduction in their sensitivity to their infants within the interaction. Their concentration on giving warm and positive feedback may make them more 'forceful' and less likely to pick up on the social bids of their infants.

While it is important to seek further support for this finding by examining interactions in more naturalistic settings and with larger samples, it seems that constraints in the attentional capacities of infants with Down syndrome may create a form of early social interaction in which the mother plays a central role in maintaining and redirecting infant attention (Landry \& Chapieski, 1989). What might be the implications of this early form of interaction for the later development of infants with Down syndrome?

\section{Social influences on cognitive development}

One possibility is that the form of interaction where the infant is dependent on the mother for engaging its attention within a dyadic social interaction may tend to 'lock in' the infant and make them less likely to spontaneously attend to objects beyond this dyad. This is supported by the findings of Landry and Chapieski (1990), who reported that 6-month-old children with Down syndrome tended to look more to their mother than an object during play. If this continues then there is a possibility that infants with Down syndrome will not engage in typical 'triadic', joint attention interactions involving mother, infant and objects with implications for later cognitive development. Triadic interactions emerge in typical infants around the end of the first year, and may be critical for the development of language, flexible thought and meta-cognitive awareness (Hobson, 2002). Even at six months atypical interactions may already have repercussions for language development (see Berger \& Cunningham, 1983). But what evidence is there that these 'forceful' interaction styles continue beyond early infancy, and does this affect the quality of triadic interactions between mothers and infants with Down syndrome? Furthermore, is there any evidence that this then impacts of the development of language and flexible thinking?

Cielinski, Vaughn, Seifer and Contreras (1995) studied children with Down syndrome of 17 to 44 months during play episodes. They measured infant engagement with toys during individual and social play and examined the quality of mother-infant interaction during the social play episodes. They found no significant group differences in comparison to typically developing children of similar developmental levels in the proportion of time infants with Down syndrome engaged with toys during individual play. However, they found significant differences in the proportion of time the infants spent engaging with toys in social play, with the children with Down syndrome spending less time engaged with toys than the typically developing children. Furthermore the children with Down syndrome spent a greater proportion of time engaging with the mother in face-toface interaction without toys.
Cielinski et al (1995) also reported that the quality of the mother-infant interactions during the social play episodes differed, with mothers of boys and girls with Down syndrome rated as more 'intrusive' and mothers of girls with Down syndrome rated as more 'directive' than mothers of typically developing girls. It was also found that maternal directiveness was positively correlated with the proportion of time the children engaged with toys for the group of children with Down syndrome but not for typically developing children. The infants with Down syndrome of more directive mothers spending more time playing with the toys. Thus, the mothers of 17-month-old infants with Down syndrome are demonstrating qualitatively different maternal behaviour in responses to their infants, compared to mothers of typically developing infants, with the mothers of infants with Down syndrome playing a more pivotal role in directing their infants' attention.

Ruskin, Kasari, Mundy and Sigman (1994) studied the attention of 22-month-old children with Down syndrome during presentations of social (a person singing) and non-social (a toy) stimuli. They reported that children with Down syndrome looked for significantly longer at the person and were more likely to engage in singing than mental age-equivalent typically developing infants. While there were no differences in attention to the toy, the infants with Down syndrome were more likely to push it away. Importantly, while this might suggest that the children with Down syndrome have relatively typical social responses with a stranger and a toy, it is important to note that the children with Down syndrome engaged in significantly less off-task looks to their mothers during this interaction, again suggesting a different quality to their joint attention with mothers. Kasari, Freeman, Mundy and Sigman (1995) also reported that compared to typically developing controls, children with Down syndrome tend to show fewer social referencing looks to their mother in an ambiguous situation, although their responses to initiated joint attention did not differ.

These results show that there is a complex relationship between an infant's attentional capacities and maternal behaviour, and that by the middle of the second year this may have evolved into a form of interaction in which mothers of children with Down syndrome act as important mediators of the infant's attention. Mothers may be compensating for constraints in capacities for endogenous attentional control by becoming more directive but also correspondingly less sensitive to the infants' bids. The findings of Ruskin et al. (1994) suggest that a possible cost of this compensatory strategy may be in the infants' subsequent inability to regulate their own behaviour in associated joint attention episodes and to spontaneously perform social referencing.

Thus, we may have an example of an earlier social factor, the nature of early mother-infant interaction, acting on the later development of spontaneous joint attention. What impact does this then have on cognitive development? While Mundy, Sigman, Kasari and Yirmiya (1988) reported 
relative strengths in social-interaction in 18-48 month old children with Down syndrome compared to mental age equivalent typically developing children, they also found a relative deficit in the tendency for children with Down syndrome to make requests for objects or for assistance with objects. Individual differences in requesting in their sample were also significantly associated with measures of expressive language. Indeed there seems to be an overall tendency for infants with Down syndrome to make fewer spontaneous social-communicative signals (Fischer, 1987). Further support for an association between early socialcommunicative capacities and language development in this population comes from a longitudinal study by Mundy, Kasari, Sigman and Ruskin (1995) which looked at children with Down syndrome when 22 and then 35 months. They found significant group differences in comparison to mental age equivalent typically developing controls in requesting and in responding to joint attention initiations from others. Also they found that individual differences in requesting and in initiating joint attention at 22 months predicted expressive language in the children with Down syndrome when 35 months old. Requesting also predicted expressive language capacities in the typically developing children but tendency to initiate joint attention did not. Mundy et al. (1995) proposed that deficits in requesting behaviour might explain expressive language deficits in children with Down syndrome and proposed that these requesting deficits may represent a primary deficit and are not a consequence of differences in affective processes or caregiver responsivity and directiveness.

However specific requesting deficits have not been found in other studies (more on this later) and, in contrast, Harris, Kasari and Sigman (1996) have reported strong associations between joint attention capacities and the development of receptive language in children with Down syndrome. While a relative strength compared to expressive language, the development of receptive language in children with Down syndrome was also found to be related to capacities for non-verbal communication. Harris et al. (1996) reported that children with Down syndrome, once engaged in joint attention will spend longer attending to caregiver selected foci of attention and that caregivers of infants with Down syndrome are more in control of the topic of attention. Critically, the best predictor of receptive language was not infant's attention to mothers' topics. Rather, receptive language was related to mothers' sensitivity to their infants' attention: mothers who more frequently redirected their attention away from the topic selected by the infant had infants who were less able in terms of receptive language.

Thus, children's attentional deficits may have a determining influence on the nature of mother-infant interaction which in turn may influence the development of expressive and receptive language. In our study, we were interested in exploring these issues taking a longitudinal perspective. We wished to establish, whether, as reported by Franco and Wishart (1995), there were differences between mental age equivalent groups of typically developing and Down syndrome infants (at 10 and 18 months), in triadic communicative acts critical for language development such as joint attention, social referencing and requesting. We also wanted to establish whether the quality of early motherinfant interaction evidenced at 6-months-of-age in infants with Down syndrome predict these differences. While the studies above provide evidence for a relationship between the quality of mother infant interaction and triadic communicative acts, they do help to determine the transactional nature and origins of this association.

In our sample we found that when the infants with Down syndrome were 18 months of age, relative to typically developing infants tested at 10 months of age, there were no specific deficits in requesting behaviour. Indeed we found some evidence for relatively spared requesting in our sample (Moore et al, 2000). In addition we did not find group differences in responses to joint attention when initiated by the tester. These findings contrast with those of Mundy et al. (1995) but mirror those of Sigman and Ruskin (1999) who, with a larger sample, reported no deficits in the initiation of requesting behaviours compared to children with other developmental delays of equivalent mental age. However, they found that the frequency of requesting and frequency of responding to joint attention was significantly less than that of mental age equivalent typically developing children. They also reported that measures of initiation and responses to joint attention were associated with measures of verbal abilities.

The reasons for the different levels of responses to joint attention in the two studies requires further exploration and may be explained in terms of matching procedures. However, although the levels of responding may have been higher for our sample, it is perhaps of more relevance that we found that within-group variation in responses to adult prompts to joint attention at 18 -months were strongly associated with the quality of mother-infant interaction (maternal 'forceful warmth') when 6-months-old. More specifically, those mothers who displayed 'forceful warmth' had infants who were less responsive to the tester's prompts for joint attention (pointing and looking at objects). This suggests that their attention is more mediated through their mother and not easily modulated by others. Interestingly this correlation also held for typically developing infants from 4 to 10 months. However, while maternal 'forceful warmth' was predicted by the infant's attention during the non-social visual attention task for the infants with Down syndrome, this was not so for typically developing infants. While similar developmental processes may be unfolding, individual differences in 'forceful warmth' of the mothers of typically developing infants may be due to factors other than their infants' attentional capacities.

\section{Conclusions}

Our findings, and those of other researchers in the field, support a transactional account of the development of infants with Down syndrome. We have proposed developmental process that lead to children with Down syndrome 


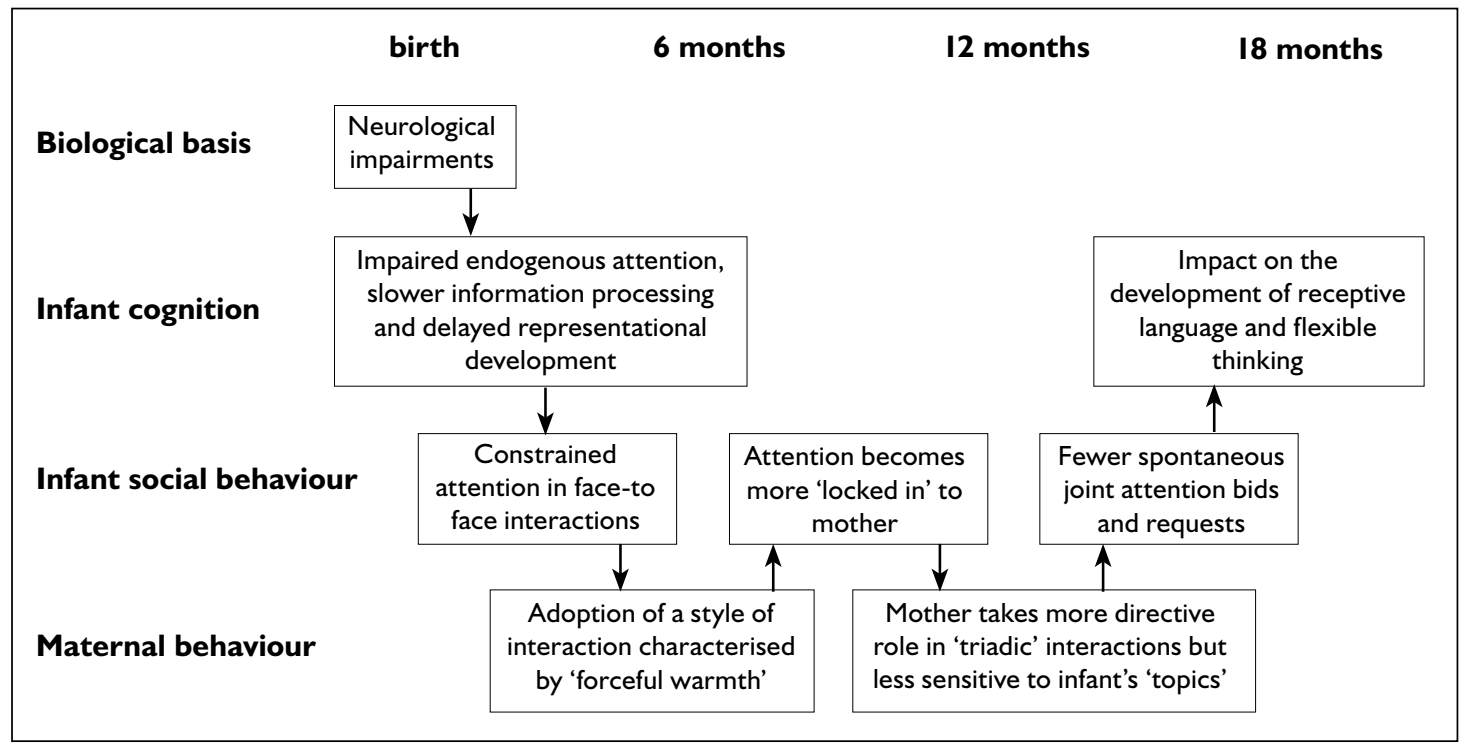

Figure I. A transactional account of the development of infants with Down syndrome

developing different patterns of triadic relations as manifest in social-communicative acts. While further work is needed to establish which social-communicative acts are most affected by the factors we have studied (i.e. requesting and/or joint attention) we can now provide a general developmental account that may underlie these deficits. A transactional model is presented in Figure 1.

We propose that neurologically-based differences in early attention regulation in children with Down syndrome may make them slower to respond and orient in social interactions. This then elicits a warmer but more forceful maternal style during interactions that serves to maintain typical levels of attention in infants with Down syndrome. This different social style may make infants with Down syndrome more focussed on the mother and may serve an important and useful function in developing early emotional attachments (Berry, Gunn \& Andrews, 1980). However, this may also make infants more 'locked in' and dependent on their mothers for regulating their attention. Subsequently, aspects of 'triadic' engagement and joint attention are more likely to be driven by the mother. Thus, when less frequent but critically important 'topic' bids are made by infants with Down syndrome, they may not be picked up because mothers are continuing to work hard to direct and maintain attention using this 'forceful' and 'warm' affective style. Ultimately, the characteristic quality of these triadic interactions may add to delays in language development and in the development of flexible symbolic thought (Hobson, 2002).

The results of our studies support an account of the development of children with Down syndrome that goes beyond simple deterministic or environmental explanations. We also offer an account that explains the development of infants with Down syndrome within the same parameters as those used to explain typical development. Of course, to confirm our hypotheses further longitudinal studies are needed, starting in early infancy and utilising additional measures and larger samples. If these studies confirm our findings, then it may be possible to provide useful suggestions for early interventions with infants with Down syndrome and their mothers, interventions that would hold promise for facilitating both social and cognitive development.

\section{Acknowledgements}

This paper is based on a keynote presentation made to the $3^{\text {rd }}$ International Conference on Language and Cognition in Down Syndrome, Portsmouth UK, September 2000. We would like to thank the mothers and infants who participated in our studies and acknowledge the ESRC for financial support (Research Grant: R000236722).

\section{Correspondence}

Derek Moore - Department of Psychology, University of East London, Romford Road, London, EI5 4LZ • E-mail: d.g.moore@uel.ac.uk

\section{References}

Bayley, N. (1969). Bayley Scales of Infant Development. New York: Psychological Corporation.

Bayley, N. (1993). Bayley Scales of Infant Development -II. New York: Psychological Corporation.

Berger, J. \& Cunningham, C. (1981). The development of eye contact between mothers and normal versus Down's syndrome infants. Developmental Psychology, 17, 678689.

Berger, J. \& Cunningham, C. (1983). Development of early vocal behaviors and interactions in Down's syndrome and nonhandicapped infant-mother pairs. Developmental Psychology, 19, 322-331.

Berger, J. \& Cunningham, C. (1986). Aspects of early social smiling by infants with Down's syndrome. Child Care, Health and Development, 12, 13-24. 
Berry, P., Gunn, P. \& Andrews, R. (1980). Behavior of Down syndrome infants in a strange situation. American Journal of Mental Deficiency, 85, 213-218.

Bruner, J. S. (1975). The ontogenesis of speech acts. Journal of Child Language, 2, 1-19.

Buckhalt, J. A., Rutherford, R. B. \& Goldberg, K. E. (1978). Verbal and nonverbal interaction of mothers with their Down's syndrome and nonretarded infants. American Journal of Mental Deficiency, 82, 337-343.

Chapman, R. S. \& Hesketh, L. J. (2000). Behavioral phenotype of individuals with Down syndrome. Mental Retardation and Developmental Disabilities Research Reviews, 6(2), 84-95.

Cielinski, K. L., Vaughn, B. E., Seifer, R. \& Contreras, J. (1995). Relations among sustained engagement during play, quality of play, and mother-child interaction in samples of children with Down syndrome and normally developing toddlers. Infant Behavior and Development, $18,163-176$.

Colombo, J. (2001). The development of visual attention in infancy. Annual Review of Psychology, 52, 337-367.

Crown, C. L., Feldstein, S., Jasnow, M. D., Beebe, B. \& Jaffe, J. (1992). Down's syndrome and infant gaze: Gaze behaviour of Down's syndrome and nondelayed infants in interactions with their mothers. Acta Paedopsychiatrica International Journal of Child and Adolescent Psychiatry, 55, 51-55.

Fischer, M. A. (1987). Mother-child interaction in preverbal children with Down syndrome. Journal of Speech and Hearing Disorders, 52, 179-190.

Franco, F. \& Wishart, J. G. (1995). Use of pointing and other gestures by young-children with Down syndrome. American Journal on Mental Retardation, 100, 160-182.

Goodwin, J. Oates, J.M. \& Moore, D.G. (2000). A short form of the BSID-II scales to assess cognitive developmental status: A pilot study with infants with and without Down's syndrome. Poster presented at the International Conference of Infant Studies, Brighton, England.

Gunn, P., Berry, P. \& Andrews, R. (1982). Looking behavior of Down syndrome infants. American Journal of Mental Deficiency, 87, 344-347.

Harris, S., Kasari, C. \& Sigman, M. D. (1996). Joint attention and language gains in children with Down syndrome. American Journal on Mental Retardation, 100, 608-619.

Heimann, M., Ullstadius, E. \& Swerlander, A. (1998). Imitation in eight young infants with Down's syndrome. Pediatric Research, 44(5), 780-784.

Hepper, P. G. \& Shahidullah, S. (1992). Habituation in normal and Down's syndrome fetuses. Special Issue: Comparative studies of prenatal learning and behaviour. Quarterly Journal of Experimental Psychology Comparative and Physiological Psychology, 44B, 305-317.

Hyche, J. K., Bakeman, R. \& Adamson, L. B. (1992). Understanding communicative cues of infants with Down syndrome: Effects of mothers' experience and infants' age. Journal of Applied Developmental Psychology, 13, 1-16.

Hobson, R. P. (2002). The Cradle of Thought: Exploring the Origins of Thinking. London: Macmillan.
Karmiloff-Smith, A. (1998). Development itself is the key to understanding developmental disorders. Trends in Cognitive Sciences, 2(10), 389.

Karrer, R., Wojtascek, Z. \& Davis, M. G. (1995). Eventrelated potentials and information-processing in infants with and without Down syndrome. American Journal on Mental Retardation, 100, 146-159.

Karrer, J. H., Karrer, R., Bloom, D., Chaney, L. \& Davis, R. (1998). Event-related brain potentials during an extended visual recognition memory task depict delayed development of cerebral inhibitory processes among 6month-old infants with Down syndrome. International Journal Of Psychophysiology, 29(2), 167-200.

Kasari, C., Freeman, S. F. N., \& Hughes, M. A. (2001). Emotion recognition by children with Down syndrome. American Journal on Mental Retardation, 106(1), 5972.

Kasari, C., Freeman, S., Mundy, P. \& Sigman, M. D. (1995). Attention regulation by children with Down syndrome -coordinated joint attention and social referencing looks. American Journal on Mental Retardation, 100, 128-136.

Landry, S. H. \& Chapieski, M. (1989). Joint attention and infant toy exploration: Effects of Down syndrome and prematurity. Child Development, 60, 103-118.

Landry, S. H., \& Chapieski, M. (1990). Joint attention of six-month-old Down syndrome and preterm infants: I. Attention to toys and mother. American Journal on Mental Retardation, 94, 488-498.

Legerstee, M. \& Bowman, T. G. (1989). The development of responses to people and a toy in infants with Down syndrome. Infant Behavior and Development, 12, 465-477.

Leslie, A. M. \& Keeble, S. (1987). Do six-month-old infants perceive causality? Cognition, 25, 265-288.

Light, P. (1986) Context, conservation and conversation. In M. Richards \& P. Light (Eds) Children of Social Worlds (pp. 170-190). Cambridge, Mass: Harvard.

Logdberg, B. \& Brun, A. (1993). Prefrontal neocortical disturbances in mental-retardation. Journal of Intellectual Disability Research, 37, 459-468.

Miranda, S. B. (1976). Visual attention in defective and highrisk infants. Merrill Palmer Quarterly, 22, 201-228.

Miranda, S. B. \& Fantz, R. L. (1973). Visual preferences of Down's syndrome and normal infants. Child Development, 44, 555-561.

Moore, D.G. (2001). Reassessing emotion recognition performance in people with mental retardation: A review. American Journal on Mental Retardation, 106, 481-502.

Moore, D.G., Hobson, R.P. \& Anderson, M. (1995). Person perception: Does it involve IQ-independent perceptual processing? Intelligence, 20 (6), 65-86.

Moore, D.G., Oates, J. Goodwin, J. \& Hobson, R.P. (2000). The regulation of visual attention in social perception, social relating and joint attention in infants with and without Down's syndrome. Presented at the International Conference of Infant Studies, Brighton, England.

Morss, J. (1983). Cognitive development in the Down's Syndrome infant: Slow or different? British Journal of Educational Psychology, 53, 40-47.

Mundy, P., Kasari, C., Sigman, M., \& Ruskin, E. (1995). Nonverbal-communication and early language-acquisition in children with Down syndrome and in normally 
developing children. Journal Of Speech And Hearing Research, 38, 157-167.

Mundy, P., Sigman, M., Kasari, C. \& Yirmiya, N. (1988) Nonverbal communication skills in Down syndrome children. Child Development, 59, 235-249.

Murray, L., Fiori-Cowley, A., Hooper, R. \& Cooper, P. (1996). The impact of postnatal depression and associated adversity on early mother-infant interactions and later infant outcomes. Child Development, 67(5), 25122526.

Oates J.M., Moore, D.G., Goodwin, J.E., Hobson, R.P. \& Reynolds, R. (1998). The behaviour of infants with Down's syndrome and their mothers in the still-face paradigm. Proceedings of the British Psychological Society, $7(2), 103$.

Oates J.M., Moore, D.G., Goodwin, J.E., Hobson, R.P. \& Reynolds, R. (2002). Developmental significance of the behaviour of mothers and their infants with Down's syndrome in the 'still-face' paradigm. Presented at the International Conference of Infant Studies, Toronto, Canada.

Oates J.M., Moore, D.G. \& Hobson, R.P. (1997). Visual attention in infants with Down's syndrome. In New Trends in Developmental Psychology: Resumé. Proceedings of the VIIIth European Developmental Psychology Conference (p.216). Rennes, France.

Piaget, J. (1954). The Construction of Reality in the Child. New York: Basic Books.

Rauh, H., Schellhas, B., Goeggerle, S. \& Muller, B. (1996). Diachronic developmental assessment of mentally handicapped young children. In M. Brambring, H. Rauh, \& A. Beelmann (Eds.), Early Childhood Intervention: Theory, Evaluation and Practice (pp. 128-154). Berlin, New York: de Gruyter.

Richard, N. B. (1986). Interaction between mothers and infants with Down syndrome: Infant characteristics. Topics in Early Childhood Special Education, 6, 54-71.

Rogoff, B. (1990). Apprenticeship in Thinking: Cognitive Development in Social Context. New York: Oxford University Press.

Ruskin, E. M., Kasari, C., Mundy, P. \& Sigman, M. (1994). Attention to people and toys during social and object mastery in children with Down syndrome. American Journal on Mental Retardation, 99, 103-111.

Sigman, M. \& Ruskin, E. (1999). Continuity and change in the social competence of children with autism, Down syndrome, and developmental delays. Monographs of the Society for Research in Child Development, Serial No 256, 64(1), 1-139.

Slater, A. (1995). Individual-differences in infancy and later IQ. Journal of Child Psychology and Psychiatry and Allied Disciplines, 36, 69-112.

Takashima, S., Becker, L. E., Armstrong, D. L. \& Chan, F. (1981). Abnormal neuronal development in the visual cortex of the human fetus and infant with Down's syndrome: A quantitative and qualitative Golgi study. Brain Research, 225, 1-21.

Tronick, E. Z., Als, H., Adamson, L., Wise, S. \& Brazelton, T.B. (1978). The infant's response to entrapment between contradictory messages in face-to-face interaction. Journal American Academy of Child Psychiatry, 17(1), 1-13.
Wertsch J.W. (1985). Vygotsky and the Social Formation of Mind. Cambridge, MA: Harvard University Press.

Wishart, J. G. \& Duffy, L. (1990). Instability of performance on cognitive tests in infants and young children with Down's Syndrome. British Journal of Educational Psychology, 60, 10-22.

Wishart, J. G. \& Pitcairn, T. K. (2000). Recognition of identity and expression in faces by children with Down syndrome. American Journal on Mental Retardation, 105(6), 466-479.

Zelazo, P. R. \& Stack, D. M. (1997). Attention and information processing in infants with Down syndrome. In J. A. Burack \& J. T. Enns (Eds.), Attention, Development and Psychopathology (pp. 123-146). New York: Guilford Press. 